\title{
LAS PRIMERAS OVARIECTOMIAS PRACTICADAS EN COLOMBIA
}

\author{
Dr. Fernando Sánchez Torres*
}

La ovariectomía fue la gran operación de una gran época en la historia de la cirugía y, particularmente, de la ginecología. Aunque parece que fue ejecutada ya en el siglo XVI por un porquero suizo en su propia hija, corresponde el mérito cierto a Efraím McDowell de haber llevado a cabo técnicamente la primera operación. Fue el 13 de diciembre de 1809, en Kentucky, cuando este cirujano norteamericano operó con feliz resultado a la señora Juana Todd Crawford. En la Gran Bretaña fue Juan Lizars quien la intentó de primero en 1823, con funestas consecuencias, y más tarde Benjamín Phillips en 1840, también con pérdida de su paciente. Dos años después Walne interviene con éxito. En 1862, The Lancet compiló setenta y nueve intervenciones de este tipo realizadas en Gran Bretaña con una mortalidad de 40 por 100.

En Alemania, al igual que en Francia, la operación fue muy impopular. Koeberlé (1862), brillante ginecólogo de Estrasburgo, fue el amo de la ovariectomía, gracias a su constancia y disciplina. Nélaton, el famoso cirujano de París, ambicionando obtener los mismos resultados de Koeberlé, hizo construir un pequeño hospital en las afueras de la ciudad para esquivar su atmósfera impura.
Sinembargo, la mortalidad de sus ovariectomizadas seguía siendo del 100 por 100 y su hospital recibió el infamante nombre de "maison du crime".

En Norteamérica se hicieron célebres con dicha intervención los hermanos Juan y Washington Atlee, de Pensilvania. El primero (1843-1883) operó setenta y ocho enfermas con setenta y cuatro supervivencias, y el segundo (1844-1878) extirpó el ovario 374 veces.

En Latinoamérica fue practicada por primera vez en Chile por el cirujano argentino Ortiz, en 1861. La segunda vez fue ejecutada en Colombia por el profesor Leoncio Barreto el 3 de octubre de 1864, en el Hospital de San Juan de Dios, de Bogotá.

Aunque en su tesis de grado "Contribución al estudio de la ovariotomía en Colombia", el doctor Eliseo Montaña consignó que la primera intervención de este tipo practicada entre nosotros fue la llevada a cabo en 1887 por los doctores Manuel Vicente de la Roche, Ramón y Francisco Arango y Juan C. Alvarez, es cierto, como bien lo advierte Bonilla Naar,

* Profesor Asistente del Departamento de Obstetricia y Ginecología de la Facultad de Medicina (Universidad Nacional). 
que en la misma tesis del doctor Montaña figura la ovariectomía que hiciera veintitrés años antes el profesor Barreto. Es probable que por no haberse abierto la cavidad peritoneal por su línea media, el doctor Montaña no la tuviera en cuenta pues él mismo anota que "aun cuando no fue una ovariotomía clásica, sí es como un preludio de ella". Pero tal vez lo que más influyó para que otorgara prioridad a la ovariectomía mencionada atrás fue la aseveración de sus autores al comunicar a la Academia de Medicina de Antioquia su hazaña quirúrgica: "Creemos - dijeron ellos-, acaso por falta de datos estadísticos, que es esta la primera vez que se practica en Colombia la ovaroitomía; y no por falta de casos, pues por el contrario los quistes $y$ demás tumores del ovario son bastante comunes entre nosotros, sino más bien por el natural temor de las enfermas de someterse a los riesgos de una operación, y sobre todo por la timidez de los cirujanos".

Escuchemos ahora el relato que de su intervención nos dejara el profesor Leoncio Barreto:

“N. R., natural de Guatavita, de 45 años, ha tenido dos hijos y presenta un grado de extenuación marcada. Sufre desde hace siete años de leucorrea y desde hace tres desórdenes marcados gastro-intestinales (vómito, náuseas, constipación, anorexia) y de la micción (incontinencia diaria, disuria nocturna). Presenta un tumor en la región ilíaca deercha del volumen de un huevo de gallina, que la exploración manual, el tacto vaginal y el rectal permiten localizar en el ovario; la operación se hizo así: el 3 de octubre se hizo una incisión, siguiendo el gran eje del tumor, después de haberlo hecho fijar por dos ayudantes; se cortó capa por capa hasta llegar al peritoneo; se hizo un ojal en éste, se seccionó con la tijera, se tiró luego el tumor hacia afuera, se colocó sobre el pedículo una ligadura en masa y se cortó por encima de ella. Los cabos de la ligadura se fijaron al exterior de la herida abdominal. Se hizo una sutura entrecortada, se colocó sobre la herida cerato, un lechino de hilas y una compresa empapada en aguardiente alcanforado. La marcha fue satisfactoria; a los catorce días cayeron los hilos de la ligadura, y a los veintisiete se levantó la enferma. En este caso se extirpó un ovario degenerado, no por una laparotomía, sino por una incisión en la región inguinal, a la manera de Houston y Laumonier". Así, dice Bonilla Naar comentando la anterior operación en su libro "Los precursores de la cirugía en Colombia", Leoncio Barreto de ignorado, pasa a ser angular en la historia nuestra y en la cirugía del continente.

La segunda ovariectomía $-y$ no "ovariotomía" como se la llama en la tesis del doctor Montaña- fue la que hizo el Dr. Manuel Vicente de la Roche, en Medellín, el 23 de julio de 1887, a una enferma de 42 años, quien había sido sometida durante largo tiempo, sin resultado ninguno, como era lógico, al tratamiento de la ergotina y del yoduro de potasio, fuera del estrujamiento a manos de un curandero. El tumo rera un cistosarcoma del ovario izquierdo que pesó dos y medio kilogramos. "La curación - dice su autor- fue completa, a pesar de las condiciones desfavorables de la enferma, y de que no fue posible cumplir con el método antiséptico, rigurosamente, por la excesiva pobreza de la enferma".

El tercer intento lo hicieron los doctores Pedro P. Estrada y Francisco Arango en Medellín, el 28 de mayo de 188. Se trataba de un carcino- 
ma de ovario con invasión abdominal generalizada. "La enferma sucumbió algún tiempo después a los progresos de la enfermedad, ya muy avanzada".

En El Poblado (fracción de Medellín) el 31 de mayo de 1888, el doctor Vespaciano Peláez junto con los doctores Julio Restrepo A. y Teodomiro Villa, extirpó un quiste multilocular voluminoso, con buenos resultados. También con la misma indicación y resultados, el doctor Pablo A. García ovariectomizó a una enferma el 21 de junio de 1888, en la ciudad de Cali. A continuación transcribimos la operación, tal como la refirió el propio cirujano durante el Segundo Congreso Médico Nacional. "Laparotomía para extirpar un gran quiste del ovario izquierdo. Junio 21 de 1888. Mujer de 35 años de edad. Ha tenido cuatro partos y dos abortos. Desde hace tres años y medio había tenido lugar el aborto anterior y desde entonces quedó abultado el vientre, abultamiento que ha seguido creciendo hasta la fecha. A la palpación hay un tumor redondo de superficie lisa ,movible, con una cintura entre sus dos tercios. Al tacto vaginal se halla el útero descendido, movible y el cuello vuelto hacia la izquierda y hacia atrás. La respiración es corta. Operación. Anestesia por el cloroformo, aseo del vientre, lavado con licor de Van Swieten tanto de la superficie externa como de las partes pudendas y vagina. Instrumentos sumergidos en solución de fenol al $5 \%$. Cirujano y ayudantes después de lavadas las manos las pasaron al bicloruro sin secarse. Incisión del ombligo al pubis. Sección de la línea blanca. Sección del peritoneo en toda la extensión de la herida. Expulsión de más o menos 100 gramos de líquido citrino. Exposición de un enorme quiste en cuya superficie se notaba una cintura. Manos mojadas en bicloruro contornearon toda su superficie y encontraron un pedículo angosto en la región del ovario izquierdo y una adherencia poco extensa al gran epiplón. Vaciado el quiste por medio del trocar de Chaisaignac dio un líquido color verde oscuro. Protección de esta sección con pinzas hemostáticas. Doble ligadura y sección de la adherencia peritoneal. Extracción del quiste y su reemplazo abdominal con una gran esponja hervida en solución de bicloruro. Aplicación de pinzas de Wells al pedículo; ligadura de éste en dos partes, aplicando otras pinzas en la inserción del pedículo alquiste y sección del pedículo. Aseo peritoneal con cuidado especial a sus divertículos. Oclusión con hilos de plata de la mitad superior de la incisión, comprendiendo en una sola sutura peritoneo, aponeurosis y piel. Extracción de la gran epsonja que abrigaba los intestinos. Terminación de la costura abdominal íntegramente. Vendaje a la gasa yodoformada, algodón y servilletas hervidas en solución de sublimado como vendaje de cuerpo. Decúbito dorsal. Extracción de la orina cada cinco horas; temperatura nocturna 38.8; pulso 120; abatimiento. Lavativas de te de buey. La temperatura continúa alta a 39.5 grados, durante los cinco días siguientes. Al séptimo día se levanta la curación, se quitan los hilos y la herida está cicatrizada. Meses después sobrevino por la cicatriz una eventración. La enferma sobrevivió 16 años".

En septiembre de 1888 y febrero de 1889, el doctor Josué Gómez, profesor de Clínica del Hospital de San Juan de Dios de Bogotá, ejecutó dos ovariectomías, ayudado por sus colegas Daniel E. Coronado, José María Buendía, Oscar Noguera, Leoncio Barreto $y$ por el entonces practicante 
de turno Luis E. García. Las intervenciones se hicieron en una pequeña sala adjunta a la del Servicio de Mujeres, llenando previamente, hasta donde era posible, las precauciones de antisepsia. En el primer caso se trataba de una formación papiliforme del ovario izquierdo, tan grande como una cabeza fetal; la enferma falleció a los cuarenta días y a la autopsia se encontró derrame purulento en la cavidad peritoneal y reincidencia del tumor en el pedículo. En el segundo caso la operada murió a las veinticuatro horas por peritonitis.

En 1889, el 11 de junio, en Jericó (Antioquia) se hizo una nueva ovariectomía, esta vez a manos de los doctores Federico A. Gómez, Rodolfo Zea y Baltasar Ochoa, con buenos resultados.

En Manizales, el doctor J. T. Henao ayudado por los doctores Velásquez y Londoño, hizo por primera vez en Colombia, y con éxito completo, una salpingo-ovariectomía doble por piosalpingitis, el 2 de junio de 1889. Al día siguiente el mismo cirujano hizo una ovariectomía bilateral por un quiste dermoide del ovario izquierdo que pesó $10 \mathrm{~kg}$. y una "degeneración marcada" del derecho. Curación.

En septiembre de 1889 el hábil cirujano Oscar A. Noguera, ayudado por el doctor José C. Güell, llevó a cabo su primera ovariectomía en Bogotá. Se trataba de un quiste multilocular del ovario derecho, muy voluminoso e intimamente adherido a las paredes abdominales, asas intestinales, vejiga, útero, estómago, hígado y bazo. Fue, al decir de Eliseo Montaña, el más difícil y complicado de todos los descritos en Colombia. La enferma evolucionó favorablemente. "Este caso - son palabras del doctor Montaña- marcará una época impor- tante en la historia de la ovariotomía en Colombia, y aun en la cirugía abdominal". El mismo doctor Noguera intentó en octubre de 1889 otra ovariectomía pero resultó ser una peritonitis tuberculosa enquistada. Al año siguiente, el 4 de junio, extirpó un fibro-cistoma voluminoso con feliz éxito.

En Bucaramanga el doctor Luis E. García, en asocio de los doctores Carlos Botero, Jesús Olaya, Daniel Cadena y Clemente Montañez, hizo la exéresis de un ovario quístico voluminoso, en noviembre de 1890, con muerte de la enferma.

En Medellín el doctor M. Uribe Angel llevó a cabo dos operaciones: una en 1888 por cistosarcoma en una mujer de 30 años, con fatal desenlace; la otra en 1891, por quiste multilocular, con buenos resultados.

Deliberadamente hemos dejado para el final la ovariectomía que practicara en Bogotá el 29 de noviembre de 1890 una de las figuras más cautivantes en la historia de nuestra cirugía: Juan Evangelista Manrique, aquel de quien dijera el maestro GuiIlermo Valencia que "entre sus manos, por ser irremediable, pudiera perderse la vida, pero nunca la esperanza".

Un día del mes de noviembre el doctor J. E. Manrique fue llamado para que examinara a doña Ignacia, esposa del que había sido su maestro en la Escuela de Medicina de Bogotá, el muy famoso doctor Antonio Vargas Vega. En junta con los profesores Joaquín Maldonado y Rafael Rocha Castilla se llegó al diagnóstico de un quiste complicado del ovario, lo cual hacía imperiosa la conducta quirúrgica, pese a todos los riesgos que ella implicaba en la entonces balbuciente época de nuestra cirugía. Pa- 
ra darnos exacta cuenta de la manera como se operaba en aquellas calendas, nada más adecuado que transcribir el relato que plasmara, en forma epistolar, la admirable pluma del escritor costumbrista don Salvador Camacho Roldán, testigo presencial y hasta actor de ese drama.

Chapinero, 1 de diciembre de 1890

Sres. Joaquín i Gabriel Camacho

Nueva York i Londres

Mis queridos hijos:

Les escribo carta en común por falta de comodidad para hacerlo por separado.

El asunto único de esta carta será la salud de Ignacia (su hermana casada con el doctor Antonio Vargas Vega): todos los demás estamos buenos.

Antier a las 9:20 a.m. empezó la terrible operación de la ovariectomía practicada en Ignacia: terminó a las 10:25, dando por resultado la extracción de un kiste -o bolsa de formación extra-normal- de 35 centímetros de largo i unos 12 ó 15 de ancho, que con su contenido representaba un peso que yo calculé en $50 \mathrm{li}$ bras a lo menos. No hubo novedad ni complicación, i la paciente lleva ya 47 horas de estado tranquilo. Anoche durmió varios ratos con entera tranquilidad, i hasta ahora según declaran los médicos, su estado es el mejor que pudiera desearse. Sin embargo, en concepto de estos hai peligro hasta el 5, día en que se le quitará el vendaje $\mathrm{i}$ algún riesgo de peritonitis hasta 25 días después, pero ya mucho menor. El $80 \%$ del mal i de los peligros de la operación ha pasado ya.

La operación fue practicada por el doctor Juan E. Manrique, en compa- ñía de los doctores Joaquín Maldonado, Rafael Rocha C., N. Güel (de Cartagena), Edward Hicks i Samuel Montaña; como practicantes funcionaron Eduardo Uribe i Eliseo Montaña, dos hermanas de la caridad, la cocinera de Ignacia i yo. Fuera de la pieza en que se operaba estaban dos criadas más, Teresita Vargas, Salustiana, Lucila i Luis Vargas, Francho Pereira, Enrique Silva i Frascisco Montaña con caballos ensillados listos para salir a buscar cualquier cosa; en la alcoba contigua se mantenía de pie un fraile franciscano (el padre Ruiz, cliente de la "Librería Colombia"), para darle la última absolución en caso de mal desenlace, i tus hermanas E. e I., Conchita, Ma. Ignacia M. i Pastora en la cocina i en el comedor de las casas contiguas preparando los almuerzos para los médicos. Todo el mundo estaba en movimiento. A Antonio $V$., cuyo carácter nervioso temíamos en ese trance, lo llevamos a una de las casas cercanas a una cuadra de distancia, en donde quedó en compañía de José Ignacio Escobar i Francisco Montaña.

La operación se hizo en el comedor de la casa de paja del cerrito de Oriente, pieza hexagonal con 6 ventanas de vidrieras, amplia, clara i perfectamente abrigada. Para levantar la temperatura a 18: centígrados, se había instalado una estufa con chimenea de lata al través del techo, i para evitar tener que salir a botar agua, también se instaló un derramadero. La temperatura se mantuvo invariablemente a 17,5 grados C., durante toda la operación. Con esa estufa y con tres grandes ollas en que constantemente se hervía agua se tuvo sin que faltara la que la operación exigió, que fue mui poco menos de un metro cúbico.

Desde el día anterior habían pasado revista los médicos al local, los 
instrumentos, la estufa, los aparatos, las 33 esponjas de todos tamaños, dos docenas de servilletas, el algodón fenicado, las bandejas i las diversas medicinas, telas, frazadas, todo desinfectado previamente con lavado en agua fenicada etc., i antes de empezar la operación se contaron de nuevo las esponjas, las pinzas etc., que debían entrar en juego, a fin de cerciorarse de que nada quedaba dentro de la cavidad abdominal.

La distribución del trabajo fue la siguiente: Operador en jefe, el Dr. Manrique; encargado de mantener la anestesia durante la operación por medio del éter, el Dr. Hicks, especialista mui práctico en este ramo; de atender al pulso de la paciente el Dr. Rocha; de cabecear las venas i arterias cortadas, el Dr. Maldonado; de servir el juego del irrigador sobre la herida, el Dr. Montaña; de ayudar a la operación quirúrgica, el Dr. Güell (que es jefe de clínica operatoria en el Hospita Ide San Juan de Dios); de alcanzar los instrumentos, el Sr. Eliseo Montaña; de atender las esponjas i servilletas calientes, Eduardo Uribe; de calentar incesantemente en un bañito especial las servilletas, una de las hermanas; de traer el agua caliente de la cocina, yo, i de mantener el fuego coke en la estufa i atender a las hermanas en la limpia de los instrumentos, baldes i platones, la criada de Ignacia. Yo ayudaba también a mantener a 37 grados centígrados el agua del aparato irrigador, observando el termómetro i echando agua caliente. Eramos 12 personas i casi no dábamos abasto al trabajo. Al terminar la operación, yo, por mi parte, estaba empapado en sudor como si hubiese estado en un baño de vapor.

La operación es terrible. Una incisión en el abdomen pone al descubierto los órganos interiores: el hí- gado, los intestinos, etc., hasta llegar al ovario: allí se separa de toda conexión con los órganos vecinos el kiste o bolsa llena de un líquido seroso, se rompe el saco i con un aparato de succión se extrae el líquido, se ligan con hilos de seda finísimos, previamente fenicados o esterilizados, los vasos y las venas que arrojan sangre por todas partes: el irrigador arroja agua a 37 ○ 38 grados centígrados incesantemente sobre la herida i las servilletas calientes cubren i mantienen además el calor dentro del hueco. En un momento no hubo más que un montón confuso de sangre $i$ de carnes vivas i estuve a pique de sentir un vértigo, pero reaccioné con la voluntad i me pasó. El kiste era multilocular, es decir, compuesto de muchos sacos pequeños como un racimo, i fue preciso reventarles a apretón de la mano del operador: eso prolongó la operación.

Durante ésta, Ignacia permanecía insensible con la acción incesante del éter que se la hacía respirar por medio de una especie de vejiga que le cubría la boca i la nariz, i que mantenía, levantándola de cinco en cinco minutos, el esterilizador. Este no levantaba la vista de su aparato i de la cara de Ignacia, aplicando el oído a cada momento para percibir el estado de la respiración, i fijo como una estatua solo atendía a su función diciendo de tiempo en tiempo "all well", "all right".

Manrique estuvo constantemente sereno sin hablar más que para pedir "chorro de agua, servilletas calientes, platón de agua para las manos", este 0 aquel instrumento, en voz baja, seca i sin emoción. En un momento dijo el Dr. Rocha "se acaba el pulso" "inyección de brandy": fue el único instante de afán. El Dr. Hicks dijo -"Be still: respiration is good"'i todo volvió a la calma. La trasla- 
ción del comedor a la alcoba estuvo algo difícil por la estrechez de tres puertas que habían de pasarse: pero Ignacia iba sobre un colchón sujeto a una tabla de 80 centímetros de anchura, con agarraderas de cinchón i pudimos pasar despacio sin comunicar movimiento alguno al cuerpo, colocando la tabla i el colchón sobre otro colchón i sábanas tendidas en la cama.

Cuando pasó la acción del éter i abrió los ojos me miró i con voz mui débil preguntó: "Antonio? Miguel José? Quiero verlos". Todavía no, dijo Manrique, espere unos instantes. Pasados estos "ya pueden venir, dijo, pero uno a uno $i$ un solo momento, sin hablar". Así se hizo: entraron Antonio, las Varguitas, José, que acababa de llegar de Bogotá, i salimos todos después.

Los peligros consistían: primero, en el éter, que puede causar asfixia. Segundo, en la posibilidad de alguna grande hemorragia. Tercero, en el choque o colapso producido por el vacío casi repentino que deja la extracción del cuerpo extraño; cuarto, en la conmoción del sistema nervioso sobre el cerebro o el corazón de una persona físicamente débil o cobarde; quinto, en la absorción de sangre extravasada o del líquido del quiste en descomposición, que puede producir envenenamiento; sexto, en algún movimiento físico, emoción moral o cosa parecida que suspenda la recomposición de los tejidos; séptimo, en alguna inflamación del peritoneo. Casi todos han pasado, solo restan los que puedan proceder de alguna infección producida por alguna pieza de vestido manchada de sangre o de líquido, de algún susto 0 emoción impensada, i de la peritonitis, que desde hoi puede empezar a aparecer. Sin embargo, hasta ahora todo va bien. Tenemos hielo incesan- temente, que suministra la señorita Ursulita Herrera, para darle pedacitos en la boca a cada momento o aplicarle al estómago i el Dr. Manrique, algún otro de los médicos y los dos practicantes, aparte de Antonio, velan incesantemente. Manrique no se apartará de la casa en cinco o seis días, a pesar de hacer un mes que se casó. Esperamos ya que Ignacia recobre completamente la salud i viva 30 años más. Hemos pasado días trabajosos, pero ya empezamos a estar tranquilos. Nadie se ha enfermado; no ha faltado nada. El Dr. Hicks dice que en Londres no se hubiera hecho la operación en mejores condiciones ni con más recursos.

Su padre que los piensa, Salvador Camacho Roldán".

Nueve días después, don Salvador escribía nuevamente a su hijo Joaquín, a París, dándole cuenta del funesto desenlace de la enfermedad de Ignacia. Oigamos algunos de los apartes de esa carta:

"Quedó Ignacia (luego de la operación) perfectamente bien, al parecer. Sin dolores algunos, dormía cortos ratos con un sueño ligero e interrumpido, i bajo la vigilancia incesante, de día i de noche, de los doctores Manrique, Güell, de los practicantes Eduardo Uribe i Eliseo Montaña, de Antonio, i con la compañía constante de las Varguitas, de tus tías Salustiana i Julia i a veces de tus hermanas, pasó los días 29 de noviembre a 6 de diciembre sin novedad alguna. El jueves 4 le examinaron el vendaje los médicos i le encontraron perfectamente cicatrizada la herida de primera intención, como ellos dicen. El 3 se le había cambiado el colchón i las sábanas i cobijas con infinitas precauciones, i se la pasó a la sala de la casa, espaciosa, abrigada i recién blanqueada con cal. 
Creían los médicos que el 8 podrían sentarse i quizás levantarse el 13 o 14, pues la juzgaban enteramente fuera de peligro. Permitieron sí, que se la alimentase ya con cucharaditas de leche con agua de cal, caldo o vino de Oporto con agua i azúcar. El 6 por la mañana prescribió ya el Dr. Manrique algunas cucharadas más de sopa de fideos i Antonio unas pocas de mazamorrita de arroz, alimento de dieta generalmente usado en Bogotá.

Desde el 4 o 5, sinembargo, había dicho Ignacia que sentía algo como frío, pero mantenía calientes los pies i la temperatura - que se le tomaba con termómetro cada tres horas- si bien subía a veces hasta $38^{\circ}$, nunca bajaba de $37^{\circ}$. El 6 fue más sensible para Ignacia la sensación de frío, i a las 3 de la tarde le principió un dolor en el bajo vientre, que fue aumentando, en términos que a las 5 le pusieron una inyección de morfina, con la cual se calmó. Sintió ganas de evacuar e hizo dos deposiciones abundantes, de materias fecales sólidas, con el auxilio de una lavativa. Pusiéronle entonces otra de aceite castor que no produjo efecto hasta 14 horas después, el 7: entre tanto siguió manifestando mucha inquietud i desazón: el calor subió a 39,5 ․ i las pulsaciones a 120 i hasta 140 por minuto: a las $11 \mathrm{p}$. m. del 6 el pulso empezó a bajar i a las 2 a.m. del 7, los médicos juzgaron que la muerte era infalible, pues le habían aplicado inyecciones subcutáneas de brandy, éter, esparteína i le habían dado digital sin éxito alguno.

La inquietud siguió aumentando i poniéndose difícil la respiración: los médicos decían que el corazón estaba paralizándose. Ella tenía la voz fuerte, se movía en la cama con facilidad i en ocasiones, sosteniéndose con los codos levantaba el cuerpo para co- ger una almohada. A la 1 p. m. expiró. Los médicos no supieron de qué. Manrique lo atribuyó a indigestión causada por la mazamorra, el Dr. Güell expresó francamente que no sabía a qu éatribuirle, Joaquín Maldonado a una hemorragia interior: los jóvenes practicantes se inclinaron a alguna reabsorción purulenta i no ha sobrado quien juzgue que fue tétano. Dicen que no hubo señales de peritonitis porque el vientre estuvo blando i no hubo vómitos sino ya horas antes de expirar. ...Me falta decirse que la conducta de los médicos ha sido ejemplar, por la consagración, el cariño y hasta pasión que mostraron por la salud de Ignacia. Los Dres. Manrique i Güell, sobre todo, merecen toda nuestra gratitud. No pudieron hacer más. Antonio reunió todos los medicamentos i aparatos con una solicitud singular, de suerte que nada faltó Sólo faltó buena suerte. Estaba escrito que debía morir en este tiempo".

Con el anterior relato, legado de un profano en cuestión de medicina, hemos pasado revista a las primeras ovariectomías practicadas en Colombia. No olvidemos que dicha intervención aquí, como en todos los países, fue el punto de partida que hizo de la ginecología una actividad netamente quirúrgica, radical y mutilante. Sobre esta histórica época el español Fernández Ruiz escribió con mucho acierto: "...no sabemos qué admirar más, si el meritorio esfuerzo y decisión de los cirujanos, a pesar de sus reiterados fracasos, y elevadísima mortalidad, o la fe y el fervor de las enfermas operadas, testimonio evidente del extraordinario prestigio de aquellos hombres que Ilenaron con sus nombres, símbolos de respeto y admiración, una época difícil y necesaria en la evolución de la disciplina". 


\section{BIBLIOGRAFIA}

1 BONILLAyNAAR, ALFONSO. "Precursores de la cirugía en Colombia". Pág. 30. Ed. Antares, Bogotá, 1954.

2 CAMACHO ROLDAN, SALVADOR. "Cartas". Repertorio Med. y Cir. 6:97, 1951.

3 DE LA ROCHE, MANUEL V.; ARANGO, RAMON; ARANGO, FRANCISCO y ALVAREZ, JUAN CLIMACO. "Operación de ovariotomía practicada con buen éxito en la ciudad de Medellín (Antioquia)". Anales de la Acad. de Med. Medellín. Año $1^{\circ} N^{0}$ 3, Pág. 108, 1888 .

4 FERNANDEZ-RUIZ, CESAR. "El camino del ginecólogo". Pág. 33. Ed. Científico Médica, Barcelona, 1956.
5 GARCIA AGUILERA, PABLO. "Evolución de la cirugía en el Cauca". Trabajo presentado al Segundo Congreso Médico Nal. Medellín, 1913.

6 LEONARDO, RICHARD. "Historia de la ginecología". Salvat Ed., S. A., Barcelona, 1948.

7 MONTAÑA, ELISEO. "Contribución al estudio de la ovariotomía en Colombia". Imprenta de Echeverría Hnos. Bogotá, 1891.

8 PEREZ, MANUEL ANTONIO. "Contribución al estudio de la cirugía abdominal en Colombia". Casa Editorial de J. J. Pérez, Bogotá, 1893.

9 ROBLEDO, EMILIO y HENAO, JOSE TOMAS. "Datos para la historia de la cirugía en el Departamento de Caldas". Trabajo presentado al Segundo Congreso Médico Nal., Medellín, 1913. 